\title{
Entender y comprender el autismo en la familia. Relato de vida de Ángel Martínez
}

\author{
Understand and understand autism in the family. Life story of Angel Martínez
}

Compreenda e compreenda o autismo na família. História de vida de Ángel Martínez

\author{
Ángel Martínez \\ angelitomart_25@hotmail.com \\ Universidad de Carabobo, Valencia, Venezuela
}

\author{
Estephanny Guaimara \\ saguaimara2410@outlook.com
}

Universidad de Carabobo, Valencia, Venezuela

\section{RESUMEN}

En el marco de la orientación existen muchas situaciones en las que no son formados los profesionales de esa área, como lo es el autismo, así que este estudio tuvo como propósito, entender y comprender el autismo en el seno de la familia desde el relato de vida de Ángel Martínez. La metodología usada fue cualitativa con enfoque biográfico específicamente relato de vida de Legrand (1993). En el análisis se produjeron ocho bloques de sentido, de los cuales se mostró de modo gestáltico dos aspectos centrales, el autismo se entiende y se comprende desde la medicina y desde la familia, como aquel seno en el cual el niño desarrolla sus talentos, aptitudes pero también como la familia misma crece, se potencia y acompaña al niño en el desarrollo de su personalidad y proyecto de vida.

Palabras clave:

Entender, comprender, relato de vida, orientación, autismo
ABSTRACT

Within the framework of guidance there are many situations in which professionals in this area are not trained, such as autism, so this study had the purpose of understanding and understanding autism within the family from the story of life of Angel Martínez. The methodology used was qualitative with a biographical approach specifically Legrand's life story (1993). In the analysis eight blocks of meaning were produced, of which two central aspects were shown in a gestalt way, autism is understood and understood from medicine and from the family, as that bosom in which the child develops his talents, aptitudes, but also as the family itself grows, empowers and accompanies the child in the development of his personality and life project.

Key words:

Understand, comprehend, life story, orientation, autism

\section{RESUMO}

No âmbito da orientação existem muitas situações em que os profissionais desta área não são formados, como é o caso do autismo, pelo que este estudo teve como objetivo compreender e compreender o autismo no seio da família a partir da história de vida de Angel Martínez. A metodologia utilizada foi qualitativa com abordagem biográfica especificamente a história de vida de Legrand (1993) .Na análise foram produzidos oito blocos de significados, dos quais dois aspectos centrais foram mostrados de forma gestáltica, o autismo é compreendido e compreendido a partir da medicina e da família, como aquele seio em que a criança desenvolve seus talentos, aptidões, mas também como a própria família cresce, capacita e acompanha a criança no desenvolvimento de sua personalidade e projeto de vida.

Palavras-chave: Compreender, compreender, história de vida, orientação, autismo 


\section{INTRODUCCIÓN}

La familia es considerada uno de los grupos primarios de mayor significación porque es el núcleo fundamental indispensable para la formación y desarrollo del ser humano, es en su seno donde se instaura el proceso de socialización, se tejen los lazos afectivos, las vivencias o experiencias del tiempo, espacio y lenguaje; así como también, donde se fomentan o generan todas las dimensiones humanas necesarias para que el hombre viva en sociedad. Entonces, es la encargada de equilibrar los problemas que sus integrantes puedan presentar en un momento dado dentro de su contexto estructural y funcional.

Por tanto, es imprescindible definir a la familia, tal como lo expresa Éroles (1997):

Es una unidad básica bio-psico-social con leyes y dinámica propias, que le permiten mantenerse en equilibrio $y$ soportar las tensiones y variaciones sin perder la identidad como grupo primario de organización social, a través de la unidad, la continuidad en el tiempo y el reconocimiento de la comunidad que lo rodea.(p. 139).

En términos generales, la familia es el sistema social por excelencia donde las personas tienen sus primeros contactos de soporte socioafectivos, primeros aprendizajes y experiencias, brindándoles las bases para el desarrollo de su futura personalidad, identidad y participación social; pues ella, como eje fundamental de la sociedad, está presente en los triunfos y fracasos del hombre, estimula o inhibe su comportamiento, y lo guía hacia el éxito.

Por lo tanto, la presencia de un miembro con autismo, dentro del núcleo familiar es una condición que origina crisis y por tanto no afecta solamente a una persona, sino a toda la familia en general; ya que necesitan de cuidados y atenciones especiales por parte de los otros miembros del grupo familiar; a tal punto que las primeras reacciones es la negación del problema, en la familia y en los padres en particular, dado a que conlleva a un duro golpe a la autoestima y la confianza en sí mismos, llegando a una profunda depresión, una sensación devastadora de desesperanza, el sentir que no están preparados para afrontar un problema de esa índole, pues es muy doloroso, desilusionante, traumático, molesto, complicado y otros muchos adjetivos que se le pueden dar a esta situación.

Así mismo Benarroch (2010), por su parte, agrega que esta afección, implica una incapacidad del individuo de responder a estímulos normales, $y$, como es de origen multifactorial, afecta muchos órganos y sistemas. Incluye desórdenes del desarrollo neurológico, compromiso del tracto gastrointestinal y una severa disfunción del sistema inmunológico.

Ante todo, esto cabe destacar que, cuando existe un miembro autista en la familia, se presentan algunos cambios en su dinámica diaria como son: agotamiento, resentimiento, responsabilidad genética, trastornos del estado de ánimo en los padres, la falta de apoyo de la familia extendida, aislamiento de la comunidad, problemas financieros, separación, divorcio, entre otros. Por tal motivo, CAIPA-SUCRE (2002) expone lo siguiente:

Cuando en la familia hay un autista no existe una relación adecuada o tal vez haya conflictos entre la pareja por el simple hecho, de que en algunos casos los padres no aceptan al niño que tiene el problema y algunos evaden su responsabilidad, incluso se presenta la separación y el divorcio; igualmente, puede presentarse una inadecuada comunicación y bajo nivel de aceptación y tolerancia, actitudes que pueden generarse como consecuencia de la falta de conocimiento e información 
que tienen para enfrentar un cuadro de autismo, lo cual, dificulta brindar apoyo a sus miembros y aún más cuando uno de ellos es autista.

De este modo, la presente investigación tuvo como propósito hacer un estudio comprensivo sobre la vivencia del entender y comprender el autismo en el seno de la familia desde el relato de vida de Ángel Martínez.

Este estudio constituye un aporte importante en el ámbito familiar y educativo, ya que permite la interacción e intercambio de experiencias desde el punto de vista historia y relatos de vida.

\section{Marco teórico}

\section{Trastorno del espectro autista}

El DSM- IV Asociación Estadounidense de Psiquiatría (2013) propone la siguiente definición:

\begin{abstract}
El autismo es una compleja discapacidad del desarrollo que aparece normalmente durante los tres primeros años de vida. Así como también, se puede considerar como el resultado de un desorden neurológico que afecta el funcionamiento del cerebro, impactando su desarrollo normal en las áreas de la interacción social y las habilidades de comunicación (s.n.).
\end{abstract}

Así mismo, el DSM-IV (2013) no etiqueta el autismo como una enfermedad sino como un trastorno, y a su vez refiere que es, el resultado de un desorden neurológico que afecta el funcionamiento del cerebro, impactando su desarrollo normal en las áreas de la interacción social y las habilidades de comunicación.

Cabe destacar que para poder diagnosticar el trastorno del espectro autista se debe cumplir con los siguientes criterios, según el DSM-IV (2013):

- Déficits persistentes en la comunicación social y la interacción social a través de múltiples contextos.
- Patrones de conductas, intereses o actividades restrictivas, repetitivas, como se manifiestan en al menos dos de los siguientes, actualmente o en el pasado.

- Los síntomas causan limitaciones significativas a nivel social, laboral u otras áreas importantes del funcionamiento actual.

\section{Teoría de la mente}

La teoría de la mente, también denominado "comprensión social" o "cognición social" se refiere a la habilidad para comprender y predecir la conducta de otras personas, sus conocimientos, sus intenciones y sus creencias. No sólo entendemos que uno mismo es capaz de tener deseos, emociones, intenciones, etc. sino que la teoría de la mente nos permite entender que los otros también tienen sus propios sentimientos, deseos, etc. (Serrat et. al., 2011)

La teoría de la mente, es la capacidad de percibir que las otras personas poseen un estado interno igual que el de un mismo y a la vez diferente de él. Esta comprensión sobre la propia mente y la mente de las personas que nos rodean, supone una herramienta importante de cara a las relaciones sociales. La teoría de la mente comporta la representación interna de los estados mentales de las otras personas.

Así que, las personas con trastorno del espectro autista (TEA) presentan dificultades para darse cuenta de lo que piensa o cree otra persona, es decir, tienen grandes problemas con la teoría de la mente. Cuando no se posee la capacidad de ponerse en el lugar de otra persona, las conductas ajenas resultan imprevisibles, sin sentido y difíciles de comprender.

La teoría de la mente permite establecer relaciones con los otros seres humanos. Gracias a esa teoría saber que existen ciertas cosas o comentarios que no se pueden decir directamente porque podrían molestar a los otros, o somos 
conscientes de que se ha dicho algo inadecuado porque se puede 'leer' las respuestas emocionales de los sujetos del entorno, entre otras cosas. No obstante, hay algunas personas que carecen de esta capacidad, como por ejemplo las personas con autismo. De forma que esto hace que sus relaciones con los otros sean muy difíciles e incluso inadecuadas.

\section{Teoría de la resiliencia}

La resiliencia distingue dos componentes: la resistencia frente a la destrucción, esto es, la capacidad de proteger la propia integridad bajo presión; por otra parte, más allá de la resistencia, la capacidad para construir un conductismo vital positivo pese a circunstancias difíciles.

Vanistendael (1994) indica que el concepto incluye la capacidad de una persona o sistema social de enfrentar adecuadamente las dificultades de una forma socialmente aceptable, así como enfatiza que cada persona es un "ser" individual, completamente único y con diferentes reacciones al momento de enfrentarse con situaciones adversas.

\section{MÉTODO}

Esta investigación estuvo enmarcada en el paradigma cualitativo, el cual es el adecuado para abordar estudios o investigaciones cuya intencionalidad fuese comprender de manera profunda realidades humanas. En este sentido, se encuentra dentro del enfoque biográfico.

El diseño de trabajo utilizado en esta investigación, recoge la propuesta hecha por Legrand (1993), sobre el relato de vida de investigación, propuesta que va desarrollando los desafíos, tareas e implicancias contenidas en cada una de las etapas de la investigación.

Cabe destacar, que las fases del relato de vida según Legrand (1993), fueron prehistoria, historia e interpretación o comprensión.
A su vez, Moreno (2006), indica sobre la validez del relato de vida, que cada historia por breve que sea, resulta ser confiable ya que cualquiera de estas contiene su carga de significados permitiendo identificarlos tanto individual como grupalmente, así pues, se encuentra estrechamente ligado a la comprensión-interpretación.

\section{RESULTADOS}

\section{Relato de vida de Ángel Martínez}

Ángel Martínez, tiene un sobrino con autismo, lo cual, para ella, como tía, ha sido una experiencia de vida única, a su vez, un cambio radical para toda la familia.

La historiadora comienza contando su historia desarrollando su vivencia con su sobrino desde hace 7 años quien tiene el trastorno del espectro autista.

Desde hace 7 años intento comprender acerca del trastorno del espectro autista, ya que tengo un sobrino que tiene dicha condición especial. He vivido la experiencia de lo difícil que es sobrellevarlo; y me refiero al comportamiento particular que el posee; muchas veces agresiva o extrema felicidad, la mayor parte del tiempo, brincando y lanzando puertas que es el juego que más le gusta.

Comprender es más que entender, se puede entender el autismo como un trastorno, causas, consecuencias y características. Pero lo que realmente Ángel expresó claramente fue "intento comprender acerca del trastorno del espectro autista" pero no lo hace, porque el comprender está muy ligado ya que tiene un sobrino en esa condición.

Así que cuando se tiene un familiar y se vive una situación desde adentro, entender no sirve; la expresión que se usa es comprender, ya que, 
comprender es ir más allá de la explicación, porque está implícito todo el sentir afectivo del sobrino, es parte de la hermana, de los padres de Ángel.

Por lo tanto, el autismo no afecta al niño sino a toda la familia; es por esto que la familia exige que cada miembro se involucre al extremo de que vayan más allá del entender, para adaptarse y superar todas las adversidades que todo esto conlleva.

Seguido, Ángel expresó, "lo difícil que es sobrellevarlo" en medio del relato, dado a que, está luchando a diario sin rendirse, entonces la protagonista no está sobrellevando sino aprendiendo a luchar. Es decir, está diciendo que se necesita un mayor esfuerzo para ayudar y tolerar cada uno de los cambios emocionales y conductuales por los que pasa el niño.

En relación a esto, la teoría de la mente acuñada por Premack y Woodruff (1978) plantea que:

Es la capacidad para comprender la existencia de estados mentales deseos, pensamientos, ideas, sentimientos entre otros; es decir la capacidad y atribuir esos estados mentales a uno mismo y a los demás, de entender que pueden ser capaz de emplear esta competencia en la predicción de situaciones derivadas del comportamiento de los demás. La teoría de la mente es crucial en el desarrollo adecuado de la condición socioemocional y el desarrollo de una conducta social competente (s.n).

Aunado a ello, la conducta del niño mostró cambios de humor drásticos, comportamientos violentos, rabietas y reacciones exageradas de alegría, mostrando inquietud y utilización de objetos inadecuados para el juego. En relación a esto el DSM-IV APA (2013) plantea el siguiente criterio; "el déficit en el desarrollo, mantenimiento y comprensión de las relaciones; que abarcan, por ejemplo, desde dificultades para ajustar su conducta para adaptarse a varios contextos sociales; dificultades para compartir el juego imaginativo". (s.n)

Así mismo, ante esta interpretación se puede resaltar lo que expresa Premack y Woodruff (1978) en la teoría de la mente donde menciona:

La mayor parte de las personas con autismo, tienen dañada la capacidad de metalización, padeciendo una especie de ceguera ante la mente de los demás e incluso la propia esta ceguera puede explicar muchos déficits que presentan a nivel social y comunicativo (s.n.).

Por lo tanto, Ángel reafirma, una vez más la resistencia y paciencia que hay que tener para poder ayudar a un hijo con autismo, aun cuando en ocasiones la hermana sienta no poder tolerar muchas situaciones, enfrenta muchas emociones para poder ayudar a su hijo. En este contexto ella reseñó:

Cabe destacar que no es fácil sobrellevarlo y no entiendo de donde mi hermana saca fuerzas para aguantar tanto, tal vez porque es su hijo, la he visto llorar hasta con ganas de no continuar.

En tal sentido, la teoría de la resiliencia según Vanistendael (1994), distingue dos componentes; "la resistencia frente a la destrucción, esto es, la capacidad de proteger la propia integridad bajo presión. Y la segunda es la capacidad para construir un conductismo vital positivo pese a las circunstancias difíciles." (s.n).

Además, Ángel señaló, que uno de los rasgos resaltantes del niño con autismo es la dificultad tardía para hablar, donde busca la manera para comunicarse a través de los gestos y señalando lo que quiere, de esta forma, expresó: 
Otra de las características que se observan en él, es que con 7 años de edad no habla y se comunica a través de señas para así dar a entender lo que desea.

Así como lo plantea el DSM-IV APA (2013) en uno de sus criterios, "el déficit en las conductas de comunicación no verbal empleadas para la interacción social, que oscilan, por ejemplo, desde una pobre integración entre la comunicación verbal y no verbal" (s.n.).

En cuanto a las dificultades físicas y cognitivas en el desarrollo del niño, Ángel reseñó, mi hermana asiste con el niño a los profesionales indicados para su orientación y ayuda: psicólogo, neurólogo, terapista conductual, terapista ocupacional y de lenguaje de esta manera se ha encaminado la estimulación y aceptación de dicha condición especial.

Ángel comentó que, para lograr el desarrollo de las capacidades del niño, debe tener la atención de especialistas adecuados y ser constantes en su asistencia a las terapias; así de esta manera, pueda alcanzar su desarrollo integral, siguiendo las orientaciones de los diferentes especialistas para tener conformidad ante esta condición especial y lo que trae consigo.

Además de la atención medica constante que debe seguir el niño, él mismo debe cumplir con una dieta estricta que le ayuda en su desarrollo, así que, Ángel expresó:

Es importante resaltar que el niño debe tener una dieta libre de gluten, trigo y caseína ya que la nutricionista recomendó esta alimentación para que su organismo no altere su digestión, conducta y habilidades cognitivas; no es fácil llevar el control de esta situación ya que tanto las consultas con médicos y cumplimiento de la dieta son costosas.

Ángel expresó, lo provechoso que es tomar en cuenta lo recomendado por la nutricionista en cuanto a la dieta del niño, puesto que es evidente que mejora la condición de su organismo, comportamiento y rendimiento cognitivo. Benarroch (2010) expresa que el no cumplir la dieta libre de gluten y caseína produce esta afectación; "la incapacidad del individuo de responder a estímulos normales, $y$, como es de origen multifactorial, afecta muchos órganos y sistemas. Incluye desórdenes del desarrollo neurológico, compromiso del tracto gastrointestinal y una severa disfunción del sistema inmunológico" (s.n).

Sin embargo, la madre ha aceptado la realidad entendiendo que lleva tiempo no solo la aceptación racional, sino también la emocional donde el amor prevalece para llevarlo todos los días a sus actividades terapéuticas y deportivas. De esta forma, Ángel, reseñó: sin embargo, sé que hay una fuerza sobrenatural para que la madre (mi hermana), se levante día a día a llevar al niño a todas las atenciones que necesita incluyendo la natación.

En relación a esto resalta Vanistendael (1994) que, la necesidad de dotar de sentido a lo que nos ocurre. Ser capaces de responder al "para qué" nos ocurren las cosas, dotándolas así de significado y aprendizaje.

Por lo tanto, Ángel reseñó:

Por medio de mi sobrino, he aprendido un poco sobre el autismo, entendí que si su dieta no se cumple el comportamiento empeora y he observado que si se le grita o maltrata se comporta peor.

En la experiencia como tía, Ángel observó los cambios positivos desde que se implementó de manera estricta la dieta en su vida, notando mejoras en su salud y en el aspecto conductual; vivenciando que se debe tener serenidad a la hora de regañar o corregir alguna conducta inadecuada. Ante esto Vanistendael (1994) menciona que, es la capacidad 
para construir un conductismo vital positivo pese a circunstancias difíciles siendo capaz la persona o sistema social de enfrentar adecuadamente las dificultades de una forma socialmente aceptable.

Ángel culminó el relato de vida, diciendo:

Aun así, ha desarrollado habilidades propias, le gusta nadar, correr, montar caballos y le fascina la tecnología, observándose feliz y tranquilo haciendo cada una de ellas.

Ángel afirmó que es importante descubrir las destrezas que posee el niño para que así pueda adquirir la independencia en cada una de ellas, disfrutando lo que hace con entusiasmo, alegría y serenidad en cada una de estas actividades. Tal cual, lo destaca Premack y Woodruff (1978) en su planteamiento donde, la comprensión del niño autista, supone una herramienta importantísima de las relaciones sociales y habilidades para la vida.

\section{CONCLUSIONES}

Surgen dos vertientes basadas en el entender y el comprender aportando así dos visiones distintas, en una primera parte, la rama de la medicina que permite entender su diagnóstico y todo lo que trae consigo y una segunda parte, basada en un enfoque humanista, el cual ayuda a visualizar la importancia de las habilidades y el apoyo familiar en la superación de situaciones difíciles.

De esta manera, el entender está ligado con la ciencia, ya que, muestra que estos comportamientos (cambios bruscos de humor, hiperactividad, uso inadecuado de objetos para jugar, entre otros), son naturales dentro del diagnóstico del trastorno del espectro autista. Y el comprender, es hacer propio una situación, es ir más profundo y conectarte con una creencia superior (el amor, la fe o incluso Dios). Siendo así el comprender lo que ayuda a percibir la situación y vivir la experiencia de manera diferente, resaltando las habilidades del niño y el apoyo familiar.

Sin embargo, el proceso es mucho más complejo de lo que a simple vista se ve, existe un entender y un comprender y que el entender esta dado mucho con el renglón del conocimiento que da la ciencia, la medicina y todas la especialidades, y que al orientador le compete abordar un poco más para que la familia se adentre en el proceso comprensivo, como el que reflejo Ángel en su relato, donde la construcción de factores, la fortaleza y el crecimiento es lo que permitió a este niño de 7 años ir superando todas sus dificultades, que no es individual, sino es todo un reto familiar.

Para finalizar, el impacto que esto tiene, es ver como en la orientación estos temas van más allá de una praxis profesional, ya que, es un asunto de vida, de vivencia comprensiva y no solo de entender las situaciones.

\section{REFERENCIAS}

American Psychiatric Association DSM IV (2013). Manual Diagnóstico y Estadístico de los trastornos mentales. Editorial Masson

Benarroch (2010). La dieta determinante para mejorar la calidad de vida de los niños con diagnóstico de autismo Recuperado el 30 de Julio del 2018 de: http://sobre-autismo. blogspot.com/2010/12/la-dieta-determinantepara-mejorar-la.html

Centro de atención integral para autista (CAIPA - SUCRE). (2002). La familia como estrategia para la optimización del proceso de atención integral de la población autista del CAIPA Sucre. Cumaná, Estado Sucre

Éroles, C. (1997). Familia y Trabajo Social. Un enfoque clínico e interdisciplinario de la intervención profesional. $1^{\circ}$ Ed. Espacio Editorial. Argentina.

Legrand M. (1993). Relatos de Vida en Enfoque biográfico. Paris: Hombres en Perspectivas. (Apuntes V. González, pp.7) Descargado el $14 / 07 / 2018$ 
Moreno, A. (2006). Al conocimiento desde la vida y su historia: Introducción teórico metodológica. Heterotopía, 32/33(1), 7-4

Premack y Woodruff (1978). La teoría de la mente. Recuperado el 30 de Julio del 2018 en: https:// bluesmarteurope.com/2013/11/30/teoria-dela-mente-y-neuronas-espejo/

Serrat, E.; Rostan, C., y Sidera, F. (2011). Llenguatge i teoria de la ment. Un estudi sobre el paper del diàleg i de l'atribució de qualitats en la comprensió de la falsa creença. Revista de Psicologia, Ciències de l'Educació i de l'Esport, 28, 121-142.

Vanistendael (1994). Teoría de la resiliencia. Recuperado el 30 de Julio del 2018 en: https://miespacioresiliente.wordpress. com/2013/03/31/la-casita-de-vanistendael/ 